\title{
BALANCING WITH POWERS OF THE LUCAS SEQUENCE OF RECURRENCE $u_{n}=A u_{n-1}-u_{n-2}$
}

\section{NURETTIN IRMAK AND MURAT ALP}

Received 30 October, 2016

Abstract. In this paper, we show that there is no solution of the diophantine equation

$$
u_{1}^{k}+u_{2}^{k}+\cdots+u_{n-1}^{k}=u_{n+1}^{l}+u_{n+2}^{l}+\cdots+u_{n+r}^{l}
$$

for special cases of $k$ and $l$ where the elements of sequence $\left\{u_{n}\right\}$ satisfy the relation $u_{n}=$ $A u_{n-1}-u_{n-2}$ with $u_{0}=0, u_{1}=1$ and $A \geq 3$ is a positive integer.

2010 Mathematics Subject Classification: 11B39

Keywords: Diophantine equation, Lucas sequence

\section{INTRODUCTION}

Let the sequence $\left\{u_{n}\right\}$ is defined by the recurrence relation

$$
u_{n}=A u_{n-1}-u_{n-2}
$$

where $A$ is a positive integer with $u_{0}=0$ and $u_{1}=1$. It's Binet form of the sequence $\left\{u_{n}\right\}$ is known as

$$
u_{n}=\frac{\alpha^{n}-\beta^{n}}{\alpha-\beta}
$$

where $\alpha=\frac{A+\sqrt{A^{2}-4}}{2}$ and $\beta=\frac{A-\sqrt{A^{2}-4}}{2}$. Assume the $\left\{v_{n}\right\}$ be the associate sequence of the sequence $\left\{u_{n}\right\}$. Namely, the elements of the sequence $\left\{v_{n}\right\}$ satisfies the following recurrence,

$$
v_{n}=A v_{n-1}-v_{n-2}
$$

with initial conditon $v_{0}=2$ and $v_{1}=A$. The Binet formula of the sequnce $\left\{v_{n}\right\}$ is

$$
v_{n}=\alpha^{n}+\beta^{n} .
$$

The case $A=6$ coincides with the sequence of balancing number (see $[2,4,7,8]$ ) whose elements satisfy the equation

$$
1+2+\cdots+(n-1)=(n+1)+(n+2)+\cdots+(n+r) .
$$


Recently, several authors handled some diophantine equations including balancingtype rules. For example, Behera et al. [3] showed that the diophantine equation

$$
F_{1}^{k}+F_{2}^{k}+\cdots+F_{n-1}^{k}=F_{n+1}^{l}+F_{n+2}^{l}+\cdots+F_{n+r}^{l}
$$

has no solution in the positive integers $n, r, k, l$ with $n \geq 2$ in the case

$$
k \leq l \text { and }(k, l)=(2,1),(3,1),(3,2) .
$$

Here $F_{n}$ denotes the $n^{\text {th }}$ Fibonacci number. They also conjectured in [3] that only the quadruple $(n, r, k, l)=(4,3,8,2)$ satisfies the equation (1.4). Their conjecture was proved by Alvarado et al. [1]. In the sequel, Irmak [5] replaced the Fibonacci numbers with balancing numbers in (1.4), and conjectured that there is no solution of the equation

$$
B_{1}^{k}+B_{2}^{k}+\cdots+B_{n-1}^{k}=B_{n+1}^{l}+B_{n+2}^{l}+\cdots+B_{n+r}^{l} .
$$

In this paper, we generalize the conjecture of Irmak [5]. Our conjecture is following,

Conjecture 1. Assume that $A \geq 3$. For the sequence $\left\{u_{n}\right\}_{n \geq 0}$, there is no solution of the equation

$$
u_{1}^{k}+u_{2}^{k}+\cdots+u_{n-1}^{k}=u_{n+1}^{l}+u_{n+2}^{l}+\cdots u_{n+r}^{l}
$$

for positive integers $n \geq 2, r, k$ and $l$.

\section{PRELIMINARIES}

In this section, we present several lemmas to confirm the conjecture. First lemma presents some formulas including the sums of the elements of the sequence $\left\{u_{n}\right\}$.

Lemma 1. For any positive integer $k$, the following identities hold.

(a) $\sum_{k=1}^{n} u_{k}=\frac{1}{A-2}\left(u_{n+1}-u_{n}-1\right)$

(b) $\sum_{k=1}^{n} u_{k}^{2}=\frac{1}{A^{2}-4}\left(u_{2 n+1}-(2 n+1)\right)$

(c) $\sum_{k=1}^{n} u_{k}^{3}=\frac{1}{A^{2}-4}\left\{\frac{u_{3 n+3}-u_{3 n}-u_{3}}{A^{3}-3 A-2}-\frac{3}{A-2}\left(u_{n+1}-u_{n}-1\right)\right\}$

(d) $\sum_{k=1}^{n} u_{2 k-1}=u_{n}^{2}$.

Proof. We prove the third one. We follow the Binet formula (1.2). Other identities can be proven by similar way.

$$
\begin{aligned}
\sum_{k=1}^{n} u_{k}^{3} & =\frac{1}{(\alpha-\beta)^{3}} \sum_{k=0}^{n-1}\left(\alpha^{k+1}-\beta^{k+1}\right)^{3} \\
& =\frac{1}{(\alpha-\beta)^{3}} \sum_{k=0}^{n-1} \alpha^{3 k+3}-\beta^{3 k+3}-3\left(\alpha^{k+1}-\beta^{k+1}\right)
\end{aligned}
$$




$$
\begin{aligned}
& =\frac{1}{(\alpha-\beta)^{3}}\left\{\alpha^{3} \frac{1-\alpha^{3 n}}{1-\alpha^{3}}-\beta^{3} \frac{1-\beta^{3 n}}{1-\beta^{3}}-3\left(\alpha \frac{1-\alpha^{n}}{1-\alpha}-\beta \frac{1-\beta^{n}}{1-\beta}\right)\right\} \\
& =\frac{1}{(\alpha-\beta)^{2}}\left\{\frac{\alpha^{3 n+3}-\beta^{3 n+3}-\left(\alpha^{3 n}-\beta^{3 n}\right)-\left(\alpha^{3}-\beta^{3}\right)}{(\alpha-\beta)\left(\alpha^{3}+\beta^{3}-2\right)}\right. \\
& \left.-\frac{3}{A-2}\left(u_{n+1}-u_{n}-1\right)\right\} \\
& =\frac{1}{A^{2}-4}\left\{\frac{u_{3 n+3}-u_{3 n}-u_{3}}{A^{3}-3 A-2}-\frac{3}{A-2}\left(u_{n+1}-u_{n}-1\right)\right\}
\end{aligned}
$$

as claimed.

Lemma 2. For positive integer $n$,

$$
u_{n}^{2}-u_{n-1}^{2}=u_{2 n-1}
$$

follows.

Proof. It can proven by the Binet formula of the sequence $\left\{u_{n}\right\}$ or Lemma $1 \mathrm{~d}$.

Lemma 3. For the positive integer $A \geq 3$ and $n \geq 1$, then the inequalities

$$
(A-2) u_{n}<u_{n+1}-u_{n}<(A-1) u_{n}
$$

and

$$
\left(A^{3}-3 A-2\right) u_{3 n}<u_{3 n+3}-u_{3 n}-u_{3}<\left(A^{3}-3 A-1\right) u_{3 n}
$$

hold.

Proof. By the Binet formulas of the sequences $\left\{u_{n}\right\}$ and $\left\{v_{n}\right\}$, we obtain the formula

$$
u_{r n}=v_{r} u_{r(n-1)}+(-1)^{r} u_{r(n-2)} .
$$

Assume that $r=3$. By the recurrence relation (1.1),

$$
\begin{aligned}
u_{3(n+1)}-u_{3 n} & =\left(A^{3}-3 A\right) u_{3 n}-u_{3(n-1)}-u_{3 n} \\
& =\left(A^{3}-3 A-1\right) u_{3 n}-u_{3(n-1)} \\
& <\left(A^{3}-3 A-1\right) u_{3 n}
\end{aligned}
$$

follows. Since the inequality $-u_{3 n}<-u_{3(n-1)}$ holds, then

$$
u_{3(n+1)}-u_{3 n}=\left(A^{3}-3 A-1\right) u_{3 n}-u_{3(n-1)}>\left(A^{3}-3 A-2\right) u_{n}
$$

follows. Second one can be proven by similar way.

Lemma 4. Suppose that $A \geq 3$. Then for all integers $n \geq 3$, the inequality

$$
\alpha^{n-1}<u_{n}<\alpha^{n-0.83}
$$

hold.

Proof. See Lemma 2.2 in [6]. 
Lemma 5. Suppose that $a>0$ and $b \geq 0$ are real numbers, and that $t_{0}$ is a positive integer. Then for all integers $t_{0} \leq t \leq t_{1}$, the inequality

$$
\alpha^{t+\kappa_{1}} \leq a \alpha^{t}+b \leq \alpha^{t+\kappa_{0}}
$$

where $\kappa_{i}=\log _{\alpha}\left(1+\frac{b}{\alpha^{t_{i}}}\right)$ for $i=0,1$.

Proof. It is obvious.

\section{Results}

In this section, we prove the several theorems which the special cases of the diophantine equation (1.6). These theorems confirm the conjecture.

Theorem 1. If $l \geq k$, then there is no solution of the diophantine equation (1.6) for positive integers $r$ and $n \geq 2$.

Proof. By Lemma 1 (a),

$$
\begin{aligned}
u_{1}^{k}+u_{2}^{k}+\cdots+u_{n-1}^{k} & <\left(u_{1}+u_{2}+\cdots+u_{n-1}\right)^{k} \\
& <\left(\frac{u_{n}-u_{n-1}-1}{A-2}\right)^{l}<u_{n}^{l}
\end{aligned}
$$

which completes the proof.

Theorem 2. If $k=2$ and $l=1$ in (1.6), then there is no solution of the diophantine equation (1.6) for positive integers $r$ and $n \geq 2$.

Proof. The Lemma 1 (a) and (b) yield that

$$
\frac{u_{2 n-1}-(2 n-1)}{A^{2}-4}=\frac{1}{A-2}\left(u_{n+r+1}-u_{n+r}-u_{n+1}+u_{n}\right)
$$

which gives

$$
u_{2 n-1}-(2 n-1)+(A+2)\left(u_{n+1}-u_{n}\right)=(A+2)\left(u_{n+r+1}-u_{n+r}\right) .
$$

Let

$$
L S:=u_{2 n-1}-(2 n-1)+(A+2)\left(u_{n+1}-u_{n}\right)
$$

and

$$
R S:=(A+2)\left(u_{n+r+1}-u_{n+r}\right) .
$$

Together with Lemma 3, we have

$$
u_{2 n-1}-(2 n-1)+\left(A^{2}-4\right) u_{n}<L S<u_{2 n-1}+(A+2)(A-1) u_{n} .
$$

So, $L S>\alpha^{2 n-2}$ holds. The Lemma 4 gives

$$
\begin{aligned}
L S & <u_{2 n-1}+(A+2)(A-1) u_{n} \\
& <\alpha^{2 n-1.83}+\alpha^{n+1.57} \\
& =\alpha^{n+1.57}\left(\alpha^{n-3.4}+1\right)<2 \alpha^{n+1.57} \alpha^{n-2.8}<\alpha^{2 n-0.5} .
\end{aligned}
$$


Similarly, we have the followings with Lemma 3

$$
(A+2)(A-2) u_{n+r}<R S<(A+2)(A-1) u_{n+r} .
$$

So, the inequalities

$$
\alpha^{n+r+0.67}<R S<\alpha^{n+r+1.57}
$$

follow by Lemma 4. Combining the inequalities (3.1) and (3.2), we deduce that

$$
\max \{2 n-2, n+r+0.67\}<\min \{n+r+1.57,2 n-0.5\}
$$

which yields that

$$
1.17<n-r<3.57 \text {. }
$$

So, there are two possibilities which are $n=r+2$ and $n=r+3$.

If $n=r+2$, then the equation (1.6) turns to the equation

$$
u_{1}^{2}+u_{2}^{2}+\cdots+u_{r+1}^{2}=u_{r+1}+u_{r+2}+\cdots+u_{2 r+2} .
$$

Above equation yields that

$$
\frac{1}{A^{2}-4}\left(u_{2 r+3}-(2 r+3)\right)=u_{r+1}+u_{r+2}+\cdots+u_{2 r+2} \text {. }
$$

Obviously, this is not possible. For the case $n=r+3$, we arrive at a contradiction similarly since we obtain the following equation.

$$
\frac{1}{A^{2}-4}\left(u_{2 r+5}-(2 r+5)\right)=u_{r+1}+u_{r+2}+\cdots+u_{2 r+3} .
$$

Therefore, we complete the proof of Theorem 2.

Theorem 3. If $k=3$ and $l=1$ in (1.6), then there is no solution of the diophantine equation (1.6) for positive integers $r$ and $n \geq 2$.

Proof. By Lemma 1 (a) and (c), the equation turns to

$$
\begin{aligned}
& \frac{1}{A-2}\left\{\frac{u_{3 n}-u_{3 n-3}-u_{3}}{A^{3}-3 A-2}-\frac{3}{A-2}\left(u_{n}-u_{n-1}-1\right)\right\}+\left(u_{n+1}-u_{n}\right) \\
& =u_{n+r+1}-u_{n+r} .
\end{aligned}
$$

Let

$$
\begin{aligned}
L S & :=\frac{1}{(A-2)\left(A^{3}-3 A-2\right)}\left(u_{3 n}-u_{3 n-3}-u_{3}\right) \\
& -\left(\frac{3}{(A-2)^{2}}+1\right)\left(u_{n}-u_{n-1}-1\right)+\frac{3}{A-2}
\end{aligned}
$$

and

$$
R S:=u_{n+r+1}-u_{n+r}
$$

We have the followings by Lemma 3 ,

$$
\frac{1}{(A-2)} u_{3 n-3}-\left(\frac{3}{(A-2)^{2}}+1\right)(A-1) u_{n-1}<L S
$$


and

$$
L S<\frac{\left(A^{3}-3 A-1\right)}{(A-2)\left(A^{3}-3 A-2\right)} u_{3 n-3}-\left(\frac{3}{(A-2)^{2}}+1\right)(A-2) u_{n-1} .
$$

Since $\alpha^{-1}<\frac{\left(A^{3}-3 A-1\right)}{(A-2)\left(A^{3}-3 A-2\right)}<\alpha^{0.63}$ and $1<\left(\frac{3}{(A-2)^{2}}+1\right)(A-2)<\alpha^{1.45}$ follow, then the inequalities

$$
\alpha^{3 n-5}-\alpha^{n-0.38}<L S<\alpha^{3 n-0.2}-\alpha^{n-1}
$$

hold which gives that

$$
\alpha^{3 n-6.5}<L S<\alpha^{3 n-0.2}
$$

By Lemma 3, we obtain

$$
(A-2) u_{n+r}<R S<(A-1) u_{n+r}
$$

which yields that

$$
\alpha^{n+r-1}<R S<\alpha^{n+r-0.17} .
$$

Together with the inequalities (3.3) and (3.4), we get that

$$
\max \{3 n-6.5, n+r-1\}<\min \{3 n-0.2, n+r-0.17\} .
$$

So there are seven possibilities which are $2 n-r=j$ where $j \in\{0,1,2,3,4,5,6\}$.

If $r=2 n$, the the diophantine equation turns the equation

$$
u_{1}^{3}+u_{2}^{3}+\cdots+u_{n-1}^{3}=u_{n+1}+u_{n+2}+\cdots+u_{3 n} .
$$

By Lemma 1 (c), we get

$$
\begin{aligned}
& \frac{1}{A^{2}-4}\left\{\frac{u_{3 n}-u_{3 n-3}-u_{3}}{A^{3}-3 A-2}-\frac{3}{A-2}\left(u_{n}-u_{n-1}-1\right)\right\} \\
& =u_{n+1}+u_{n+2}+\cdots+u_{3 n} .
\end{aligned}
$$

Since $\alpha^{-5}<\frac{1}{\left(A^{2}-4\right)\left(A^{3}-3 A-2\right)}<\alpha^{-3.26}$ holds, then $\alpha^{3 n-6}<\frac{u_{3 n}}{\left(A^{2}-4\right)\left(A^{3}-3 A-2\right)}<$ $\alpha^{3 n-4.09}$ follows. But this is not possible since the inequality $\alpha^{3 n-1}<u_{3 n}<\alpha^{3 n-0.83}$ holds. Since we have similar calculations for the cases $j \in\{1,2,3,4,5,6\}$, we omit the proofs of these cases to cut unnecessary calculations. So, we complete the proof of Theorem 3.

Theorem 4. If $k=3$ and $l=2$ in (1.6), then there is no solution of the diophantine equation (1.6) for positive integers $r$ and $n \geq 2$.

Proof. By Lemma 1 (b) and (c), we have

$$
\begin{aligned}
& \frac{u_{3 n}-u_{3 n-3}-u_{3}}{A^{3}-3 A-2}-\frac{3}{A-2}\left(u_{n}-u_{n-1}-1\right)+u_{2 n+1} \\
& =u_{2(n+r)+1}-2 r .
\end{aligned}
$$


Let

$$
R S:=u_{2(n+r)+1}-2 r
$$

and

$$
L S:=\frac{u_{3 n}-u_{3 n-3}-u_{3}}{A^{3}-3 A-2}-\frac{3}{A-2}\left(u_{n}-u_{n-1}-1\right)+u_{2 n+1} .
$$

Together with Lemma 4, we have

$$
\alpha^{2(n+r)}-2 r<R S<\alpha^{2(n+r)+1-0.83} .
$$

The inequalities

$$
\begin{gathered}
\alpha^{3 n-4}<u_{3 n-3}<\frac{u_{3 n}-u_{3 n-3}-u_{3}}{A^{3}-3 A-2}<\frac{A^{2}-3 A-1}{A^{2}-3 A-2} u_{3 n-3}<\alpha^{3 n-3.89}, \\
\alpha^{n-2}<3 u_{n-1}<\frac{3}{A-2}\left(u_{n}-u_{n-1}-1\right)<\frac{3(A-1)}{A-2} u_{n-1}<\alpha^{n-1.83+1.15}
\end{gathered}
$$

and

$$
\alpha^{2 n}<u_{2 n+1}<\alpha^{2 n+0.17}
$$

yield that

$$
\begin{aligned}
& \alpha^{n-0.68}\left(\alpha^{2 n-3.32}-1+\alpha^{n+0.68}\right) \\
& =\alpha^{3 n-4}-\alpha^{n-0.68}+\alpha^{2 n}<L S \\
& <\alpha^{3 n-3.89}-\alpha^{n-2}+\alpha^{2 n+0.17} \\
& =\alpha^{n-2}\left(\alpha^{2 n-2.89}-1+\alpha^{n+2.17}\right) .
\end{aligned}
$$

Since $\alpha^{2 n-4.1}<\alpha^{2 n-3.32}-1$ and $\alpha^{2 n-2.89}-1<\alpha^{2 n-2.88}$ hold, then we obtain

$$
\alpha^{n-0.68}\left(\alpha^{2 n-4.1}+\alpha^{n+0.68}\right)<L S<\alpha^{n-2}\left(\alpha^{2 n-2.88}+\alpha^{n+2.17}\right) .
$$

By the inequalities $\alpha^{2 n} \alpha^{n-4.78}<\alpha^{n-0.68}\left(\alpha^{n+0.68}\left(\alpha^{n-4.78}+1\right)\right)=$ $\alpha^{n-0.68}\left(\alpha^{2 n-4.1}+\alpha^{n+0.68}\right) \quad$ and $\quad \alpha^{n-2}\left(\alpha^{2 n-2.89}+\alpha^{n+2.17}\right)=$ $\alpha^{n-2}\left(\alpha^{n+2.17}\left(\alpha^{n-5.06}+1\right)\right)<\alpha^{n-2+n+2.17+n-1.9}$, then

$$
\alpha^{3 n-4.78}<L S<\alpha^{3 n-1.73}
$$

follows. Together with inequalities (3.5) and (3.6), the condition

$$
\max \{3 n-4.78,2 n+2 r\}<\min \{3 n-1.73,2 n+2 r+0.17\}
$$

gives that $1.73<n-2 r<4.95$. So, the possible cases are $n-2 r=i$ where $i \in$ $\{2,3,4\}$. When we replace $n=2 r+i$ in the equation (1.6), we get that

$$
u_{1}^{3}+u_{2}^{3}+\cdots u_{2 r+i-1}^{3}=u_{2 n+2}^{2}+u_{2 n+3}^{2}+\cdots+u_{3 r+i}^{2} .
$$

Obviously, the left hand side is less than the right hand side. Therefore, we arrive at a contradiction. Hence, the proof is completed. 


\section{REFERENCES}

[1] S. D. Alvarado, A. Dujella, and F. Luca, "On a conjecture regarding balancing with powers of Fibonacci numbers." Integers, vol. 12, no. 6, pp. 1127-1158, a2, 2012, doi: 10.1515/integers-20120032.

[2] A. Behera and G. Panda, "On the square roots of triangular numbers." Fibonacci Q., vol. 37, no. 2, pp. 98-105, 1999.

[3] A. Behera, K. Liptai, G. K. Panda, and L. Szalay, "Balancing with Fibonacci powers." Fibonacci $Q .$, vol. 49, no. 1, pp. 28-33, 2011.

[4] R. Finkelstein, “The house problem.” Am. Math. Mon., vol. 72, pp. 1082-1088, 1965, doi: $10.2307 / 2315953$.

[5] N. Irmak, "Balancing with balancing powers." Miskolc Math. Notes, vol. 14, no. 3, pp. 951-957, 2013.

[6] N. Irmak and L. Szalay, "Diophantine triples and reduced quadruples with the Lucas sequence of recurrence $u_{n}=A u_{n-1}-u_{n-2}$." Glas. Mat., III. Ser., vol. 49, no. 2, pp. 303-312, 2014, doi: 10.3336/gm.49.2.05.

[7] G. Panda, "Sequence balancing and cobalancing numbers." Fibonacci Q., vol. 45, no. 3, pp. 265271, 2007.

[8] G. Panda, "Some fascinating properties of balancing numbers." Congr. Numerantium, vol. 194, pp. 185-189, 2009.

Authors' addresses

Nurettin Irmak

Niğde Ömer Halisdemir University, Art and Science Faculty, Mathematics Department, 51240, Niğde, Turkey

E-mail address: nirmak@ohu.edu.tr, irmaknurettin@gmail.com

Murat Alp

Niğde Ömer Halisdemir University, Art and Science Faculty, Mathematics Department, 51240, Niğde, Turkey

E-mail address: muratalp@ohu.edu.tr 